\title{
Simulating vegetation in ancient Japan using HUMPOL: A pollen-based multi- scenario modeling approach
}

\author{
Lauren Bell1', E. Crema², M.J. Bunting ${ }^{3}$ and M. Madella ${ }^{4}$
}

\section{We present a hypothetical vegetation map for the largest Jōmon settlement in Japan (ca. 5900-4100 BP) based on simulated pollen dispersal in the landscape using fossil pollen data. It suggests intentional planting of chestnut groves within the settlement area.}

The simulation of vegetation in past landscapes can be important for archaeologists attempting to reconstruct ancient land-use and human-induced land-cover changes. This research focuses on the application of the software HUMPOL (Bunting et al. 2004), a modeling suite based on the "Prentice-Sugita model" from the REVEALS-LOVE algorithm of pollen dispersal and deposition in lakes and bogs similar to the approach used in POLLANDCAL (Sugita 2007a, b; Sugita 1994; Sugita et al. 1999). However, HUMPOL expands on this by enabling the testing of multiple vegetational hypotheses on known landscape parameters, known as the Multi-Scenario Approach (MSA; Bunting and Middleton 2009). HUMPOL has been used in Europe to determine the extent of human impacts on past vegetational landscapes (e.g. Caseldine and Fyfe 2006; Gaillard et al. 2008). Models can provide insight into patterns otherwise invisible in the archaeological record. They are particularly useful when studying large areas with multiple data sources. HUMPOL is a valuable tool with which the user can test hypotheses on past landscapes by altering multifarious environmental parameters and variables (e.g. degree of vegetational openness) to assess their effect on vegetation cover

\section{Using HUMPOL for vegetation \\ reconstruction}

HUMPOL is most effectively used for vegetation reconstructions at local to sub-regional spatial scales, particularly in projects with good pollen core coverage and a robust chronology for the relevant time slices. To successfully define landscape parameters for the input map, we must assume the dominant pollen taxa reflect the different vegetation/ landscape/land-use types in their ecological system. Pollen dispersal is simulated using this input map.

One major prerequisite for HUMPOL input grid creation is landscape data, most crucially on elevation and hydrology. Geology and soil information can also be included. Unfortunately, as not all spatial data can be placed directly into the HUMPOL software suite, preparation of the data is usually one of the first stages in the modeling process (Fig. 1). The elevation data can be obtained from a high resolution Digital Elevation Model (DEM).

When creating the simulated landscapes, each selected pollen taxon and its preferences in terms of landscape parameters will affect where the pollen will be deposited on the grid, and determine its abundance once it reaches the plant's ideal habitat. For this, the ecological preferences of each plant taxon used in the study need to be known, such as tolerance to slope, facing direction of the slope, and wetness (Fig. 1). To simulate pollen deposition in the pre-defined landscape, HUMPOL also requires pollen productivity estimates (PPE) and fall speed values for each taxon. Pollen productivity differs between plant taxa: for instance, trees generally produce more pollen than herbs. Furthermore, size and shape differs between pollen-morphological types, which affects their aerodynamics and thus the speed at which they are transported by wind and the distance they travel. PPEs are generally obtained empirically using field data, i.e. datasets of modern pollen assemblages from multiple pollen traps across a specific ecological system (e.g. Mazier et al. 2012). Fall speed is calculated based on measurements of pollen grains for each plant taxon on the basis of Stoke's Law, which describes fall speed of small particles in the air (see also Dawson et al., this issue). Once pollen assemblages are simulated for the hypothetical landscape, they can be compared to the fossil pollen record (Fig. 1).

HUMPOL at Sannai Maruyama secondary forest or chestnut gardens? Sannai Maruyama is situated on a hilltop surrounded by valleys which lead into the Okidate River in modern-day downtown Aomori City in northern Japan (Fig. 2a), and is one of the largest Jōmon settlements known to date. Settled from ca. 5900-4100 BP, Sannai Maruyama displays evidence of pit dwellings, mounds, pottery, clay figurines, and stone implements (Kawahata et al. 2009; Tsuji and Nakamura 2001). Environmental data obtained from waterlogged areas suggest that during occupation there was a prevalence of chestnut trees around the site, a feature rarely seen in Jōmon sites. As chestnut (Castanea crenata Siebold \& Zucc.) is a sun-loving, insect-pollinated tree,

1. Input grid creation

3. Simulation model creation

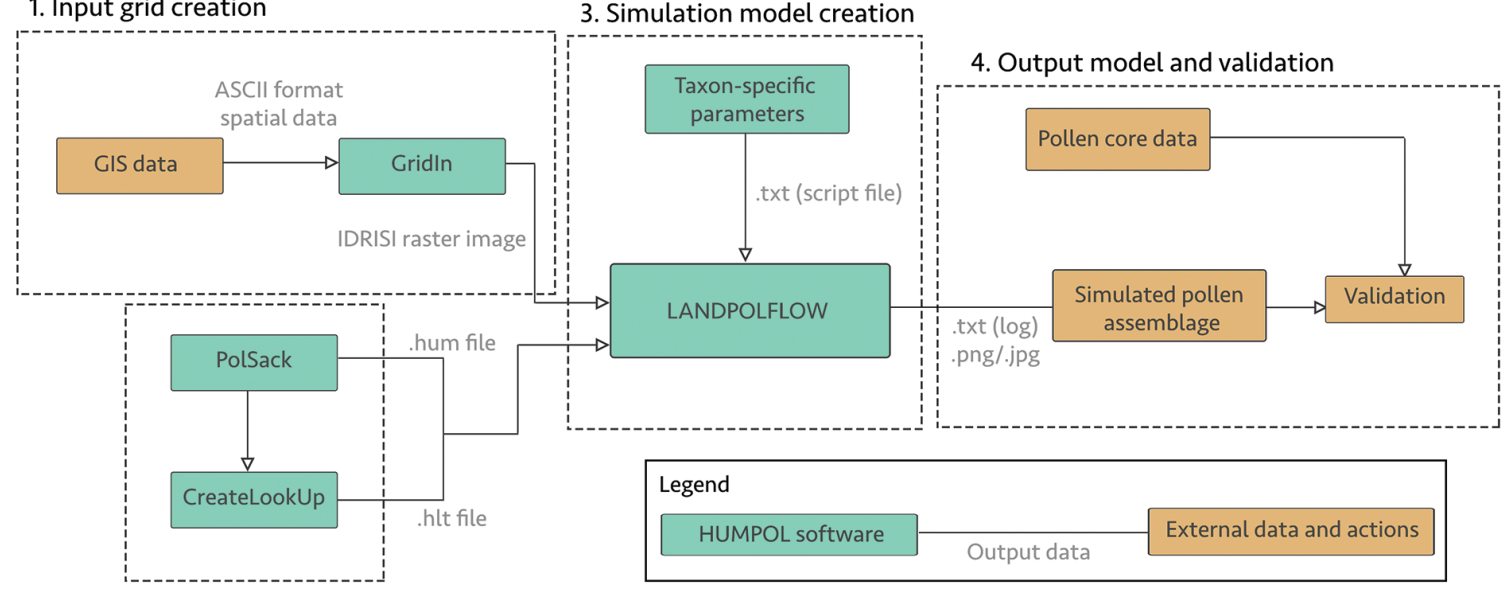

2. Taxon and community data

Figure 1: A workflow for the HUMPOL software suite. Gridln converts ASCII-format spatial data into the IDRISI-format grid with a specified cell size. PolSack creates unique identifiers and allows input of data for each taxon, community, and pollen core. CreateLookUp creates a distance-weighted vegetation lookup table based on abundance and location of each taxon in the landscape using the Prentice-Sugita model. Script file (.txt) contains all programmable instructions for the landscape parameters and MSA variables. LANDPOLFLOW simulates the dispersal and deposition of pollen in the hypothetical landscape based on data from all the above. 
it naturally grows sparsely in valley settings. Human intervention therefore seems the only possibility for expanding its presence (Yoshikawa 2011). The purpose of this research was to test whether climate, passive human presence, or active management of the landscape could be identified as key factors for chestnut's expansion at this time.

Five pollen cores from adjacent valleys were selected due to their reliable dating to around $5100 \mathrm{cal}$. BP, their spread across the site, and sufficient representation of key dominant taxa. As geology and soil characteristics did not vary enough to have had an effect on vegetation, only a DEM of the area $\left(1.4 \mathrm{~km}^{2}\right)$ and a reconstructed hydrological map were used. PPEs and fall-speed values were acquired from studies in Europe (Mazier et al. 2012) and Japan (Bunting, pers. comm.). To assess the scale of human impact on the vegetation at Sannai Maruyama, two landscape scenarios were created in HUMPOL from two time slices: (1) pristine natural vegetation before the establishment of the settlement; and (2) anthropic vegetation at the time of peak settlement occupation. In the second scenario, three main factors were accounted for. As climate was warming around $5100 \mathrm{cal}$. BP, the effects of this on the pollen record were explored by increasing the abundance of sun-loving species, Castanea, Juglans and Aesculus. The presence of a secondary forest, as hypothesized by Nishida (1983), was represented in the simulation as a community of taxa associated with human exploitation around the settlement areas. To simulate the effects of intentional fires, variables for landscape openness were used in the settlement areas and in the secondary forest.

The results showed that it is possible to attain a close match to the pollen record at Sannai Maruyama and reproduce some of the expected patterns based on hypothesized anthropic effects on the vegetation (Fig. 2b). Because it was difficult to replicate the values of Castanea and Gramineae, it can be suggested that Jōmon management methods involved transplanting chestnut trees into the settlement area from saplings collected from the valleys. This disproves Nishida's (1983) hypothesis regarding the layout of the secondary forests. If the modeled hypothetical landscape is correct, it would imply that the areas with pit dwellings were lined with chestnut groves whose produce was shared within the community. However, the HUMPOL software suite is limited in its representation of insectpollinated plant taxa, such as Castanea. This is because the Prentice-Sugita model assumes that all pollen is dispersed by wind; therefore, the simulated pollen assemblages might be misleading. Nevertheless, using HUMPOL for this project has been very useful as an exercise in assessing the effects of potential causes of vegetation changes in the landscape, although further exploration is needed to reach more definitive conclusions. More reliable dates for the other existing pollen records from Sannai Maruyama, for example, would improve the resolution of the models produced.

Comparisons to other similar settlements with good pollen-core coverage could also be valuable for furthering this research.

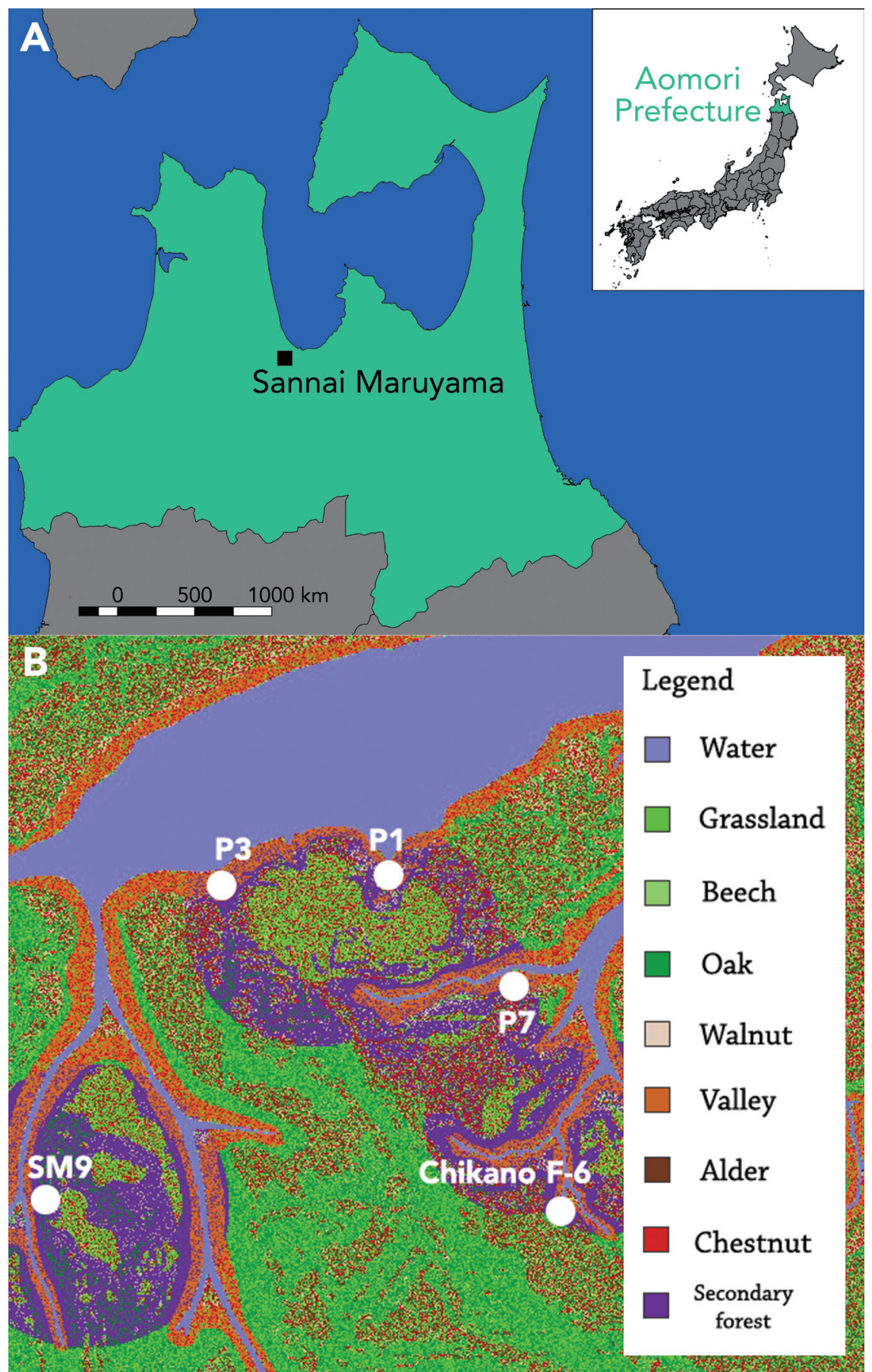

Figure 2: (A) Map of Sannai Maruyama within Aomori Prefecture; (B) The output map from LANDPOLFLOW showing the hypothetical vegetation for the anthropic model $\left(1.4 \mathrm{~km}^{2}\right)$. This map resulted in the simulated pollen assemblages with the best statistical fit for the fossil pollen assemblages from the five pollen records P1, P3, P7, Chikano F6 and SM9 (white dots).

\section{ACKNOWLEDGEMENTS}

This paper is based on a dissertation undertaken for a BSc in Archaeology at University College London (UCL). With thanks to Prof. Junko Habu for helping to start this project, and Prof. Mark Lake for supervising the dissertation.

\section{AFFILIATIONS}

${ }^{1}$ School of Archaeology, University of Oxford, UK ${ }^{2}$ Department of Archaeology, University of Cambridge, UK

${ }^{3}$ Department of Geography, University of Hull, UK ${ }^{4}$ Department of Humanities (CaSEs, ICREA), University Pompeu Fabra, Barcelona, Spain

CONTACT

Lauren Bell: lauren.bell@hmc.ox.ac.uk

\section{REFERENCES}

Bunting MJ et al. (2004) Holocene 14: 651-660

Bunting MJ, Middleton R (2009) Holocene 19: 799-803
Caseldine C, Fyfe R (2006) Quat Sci Rev 25: 632-644 Gaillard MJ et al. (2008) Veg Hist Archaeobot 17: 419-443 Kawahata H et al. (2009) Quat Sci Rev 28: 964-974

Mazier F et al. (2012) Rev Palaeobot Palynol 187: 38-49 Nishida M (1983) J Anthropol Archaeol 2: 305-322 Sugita S (1994) J Ecol 82: 881-897 Sugita S et al. (1999) Holocene 9: 409-421 Sugita S (2007a) Holocene 17: 229-241 Sugita S (2007b) Holocene 17: 243-257 Tsuji S, Nakamura T (2001) Quat Res 40: 471-484 Yoshikawa M (2011) Jap J Hist Bot 18: 65-76 